\title{
Some aspects of collagen maturation and fibrosis
}

\author{
ALLEN J. BAILEY \\ From the Agricultural Research Council, Langford, Bristol
}

In the first paper (page 2) Professor D. S. Jackson reviewed the biosynthesis of the collagen molecule. This paper discusses the subsequent extracellular events - that is, the formation of a collagen fibre, the production of intermolecular cross-links to provide the fibre with mechanical strength, and the age-related changes in the properties of the fibre. These developmental changes up to maturation are then compared with those occurring in pathological fibrosis.

After secretion of the procollagen molecule the extension peptides are removed and the resultant collagen molecule is incorporated into fibres under physiological conditions. The exact point of cleavage has not yet been clearly defined, and possibly it is a multistep process in which each discrete stage has some function in the control of fibrogenesis-for example, the cleavage of the $\mathrm{N}$ - and $\mathrm{C}$-terminal peptides could involve separate enzymes which may be highly tissue specific and the rate and extent of peptide cleavage could have a control function in fibrillogenesis.

At present four genetically distinct types of triplehelical collagen molecules have been identified (Miller and Matukas, 1974) and it is more than likely that further types will be distinguished. In particular type IV, which has not yet been fully characterized, may be a mixture of distinct types (Daniels and Chu, 1975).

It is generally accepted that the molecules in the newly formed fibre are aligned in parallel, each molecule being quarter-staggered with respect to its neighbour, and the ends of the molecules overlapped (Hodge and Petruska, 1963). However, the precise details of the three-dimensional arrangement of the molecules have not yet been worked out, although a number of models have been proposed (Miller and Wray, 1971; Hoseman et al., 1974; WoodheadGalloway et al., 1975). Collagen types I to III all form collagen fibres with the typical $68-\mathrm{nm}$ axial periodicity (Weidmann et al., 1975). Basement membrane collagen, or type IV, however, does not form fibres. The molecules aggregate to form an almost amorphous membrane, the faintly discernible fibrils lacking the typical banding of collagen (Clark et al., 1975).

The high tensile strength of the collagen fibre is due to the presence of covalent cross-links between the collagen molecules making up the fibre. In the absence of these cross-links, as in experimental lathyrism, the fibres are extremely fragile and are क readily soluble in neutral salt buffers (Levene and $\overrightarrow{0}$ Gross, 1959). This is true of the collagen fibres from types I and II but type III fibres remain insoluble and $\vec{\omega}$ require extensive pepsin treatment to solubilize them.

The chemistry of the cross-links has recently been $\omega_{\text {o }}$ reviewed (Bailey et al., 1974) and I will therefore $\omega$ limit my comments to data pertinent to the changes in the cross-links during development and in fibrotic situations.

\section{Development}

The first stage in the biosynthesis of the cross-links involves the enzyme lysyl oxidase, which oxidatively deaminates specific lysyl and hydroxylysyl residues located in the non-helical $\mathrm{N}$ - and C-termin\&l regions of the collagen molecule. The aldehydes formed then condense spontaneously with the $\varepsilon-\mathrm{NH}_{2}$ of a hydroxylysine within the triple helicay body of an adjacent molecule, thus forming an intermolecular bond. The structure of these crosslinks has been elucidated by characterization of ${ }_{0}^{\circ}$ their borohydride-reduced forms. Based on these $\stackrel{\unrhd}{\complement}$ structures it was concluded that both cross-links $\overrightarrow{\vec{O}}$ existed as aldimine bonds in vivo (I and II), but the 3 unusual stability of one of these aldimine bonds (II) has now been shown to be due to an Amadorio rearrangement-that is, migration of the double bond to produce the more stable keto form III.? The structure of these cross-links is as follows:
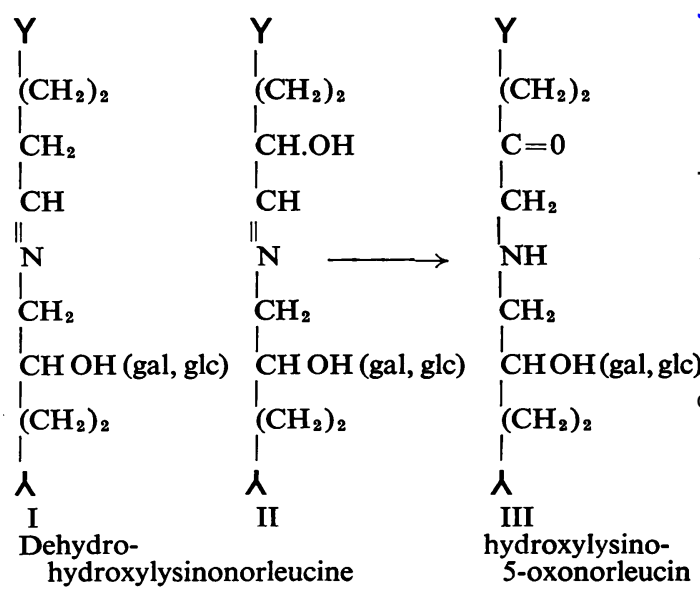
Both types of cross-link are intermolecular covalent bonds and as such confer a high tensile strength on the fibre. However, those tissues containing a high proportion of the aldimine bond (I)for example, skin and rat tail tendon-tend to be more soluble in acid buffers and on denaturation, whereas those possessing a high proportion of the keto bond (III) (for example, bone and cartilage) are highly insoluble.

The high proportion of type III collagen in early fetal skin compared to adult skin (Epstein, 1974) initially suggested that it should be considered an embryonic collagen, but high proportions have now been shown to be present in other adult tissues-for example, aorta (Rauterberg and von Bassewitz, 1975), viscera (Epstein and Munderloh, 1975), and synovial membrane (Eyre and Muir, 1975; Lovell et al., 1976). It is highly likely, therefore, that type III collagen performs a specific functional role in these tissues.

The rapid postnatal replacement of the type III collagen molecules by type I closely parallels the change over in the nature of the cross-links (Bailey et al., 1974). However, detailed analysis showed that both types I and III of embryonic dermal collagen were stabilized by hydroxylysino-5-keto-norleucine and that postnatally both types possessed the aldimine cross-link, dehydrohydroxylysinonorleucine (Bailey and Sims, 1976). Thus both types of molecules possess hydroxylysine in the telopeptides when present in embryonic tissues but revert to lysine in collagen synthesized by adult tissues. These results are consistent with the overall increased hydroxylation of embryonic collagen (Barnes et al., 1974), although it is noteworthy that the increased hydroxylation of the telopeptide lysine involved in cross-linking is proportionately greater than those in the helical body of the molecule.

\section{Maturation}

The formation of a fibre stabilized by the above cross-links may appear to complete the biosynthesis. However, further changes in the physical properties of collagen with age are well established. The fibres increase in tensile strength, become less soluble, and more resistant to chemical and enzymic attack. These changes are indicative of alterations in the extent and nature of the intermolecular crosslinking (Bailey and Robins, 1973).

Analysis of tissues for the reducible cross-links showed that as the growth rate slowed down so the amount of these cross-links decreased, until at maturity they were barely detectable. This suggests that the reducible aldimine cross-links are stabilized during maturation to a non-reducible form (Robins et al., 1973). The conversion to a stable cross-link would readily account for the age-related changes in the physical properties of dermal collagen. Recently proposals have been made as to the nature of these stable cross-links but more experimental evidence is required to confirm these structures (Davis et al., 1975; Hunsley, 1975).

In summary, considerable changes have been shown to occur in the collagen from fetus to senescence. The changes in the dermal collagen are of particular interest. The change over from type III to type I collagen is accompanied by a change from the keto to the aldimine cross-links in both types of collagen. Subsequently the labile aldimine bonds are stabilized by the formation of as yet unknown nonreducible cross-links during maturation. At present the physiological significance of these changes is unknown and a more detailed study of the effect of different types of cross-links on the properties of the native fibre would provide better understanding of this complex process.

\section{Fibrosis}

Many common debilitating diseases involve the proliferation of collagen. It is therefore of considerable importance to investigate the rapid proliferation of collagen in mature tissues and compare the findings with the changes known to occur during normal development. In some situations there is an obvious synthesis of new collagen-for example, granulation tissue in wound healing-while in others it is not clear whether there is an increase in synthesis or a decrease in the rate of degradationfor example, scleroderma.

Newly synthesized collagen in mature tissues should be readily detectable by the presence of the intermediate reducible cross-links. We have used this technique to demonstrate the proliferation of collagen in dermal scleroderma (Herbert et al., 1974), intervertebral discs (Herbert et al., 1975), and rheumatoid synovium (Lovell et al., 1976). These results will be discussed in detail in later papers in this symposium.

Recently we have studied the collagen of granulation tissue produced in response to injury. Acute and chronic inflammation produced by subcutaneous implantation of turpentine and polyvinyl sponges respectively have been used as models for wound healing. The collagen of the granulation tissue contained a higher proportion of type III collagen than the surrounding skin and in contrast to the aldimine cross-link of normal skin both the type I and III collagens possessed the stable keto crosslink. During healing the proportion of type III and the type of cross-link reverted to that observed in normal skin. In contrast, with long-term sponge implants the synthesis of type III is maintained and the cross-links do not revert to those of normal skin (Bailey et al., 1973; Bailey et al., 1975a). 
A similar sequence of events has now been shown to take place with normal and hypertrophic human scars (Bailey et al., 1975b). The collagen of normal scars initially contains a high proportion of type III collagen and is stabilized by the keto cross-link, but subsequently undergoes the same age-related changes observed in normal skin. Similar results have also been obtained with normal scars from guinea pigs (Barnes et al., 1976), although other workers, using open-splinted wounds, failed to detect type III (Shuttleworth et al., 1975). Hypertrophic scars, on the other hand, followed a similar pattern to the sponge implants in retaining characteristics of embryonic collagen, no normal maturation effects being observed even with hypertrophic scars of many years standing (Bailey et al., 1975b).

In short, clearly the changes in the collagen of granulation tissue during wound healing are closely analogous to those occurring during normal development. However, in neither case can the significance of these changes in genetic type of collagen and stabilizing cross-link be related to the changes in the functional requirements of the tissue.

The fibroblasts in early wound healing and in experimental granulation tissue have been shown to be immature fibroblasts developing a contractile apparatus which make them similar to smooth muscle cells (Gabbiani et al., 1972). Whether this represents a conversion in cell type or cell selection remains to be seen. Possibly the initial stimulus to produce collagen results in the formation of an embryonic collagen from these cells. In normal scars the metabolic activity of the local fibroblasts is strictly controlled but in hypertrophic scars it is clearly disturbed. In other fibrotic situations the course of events may be different-for example, the dermal collagen produced in the sclerodermatous plaque, in rheumatoid synovial membrane, and in thickened basement membrane in diabetes appears in each case to produce an excess of an apparently normal collagen. The response to injury may well depend on the nature of the stimulating factor. Macrophages have often been implicated in activating the proliferation of connective tissue (Carr, 1973) and obviously deserve a closer study in this respect.

The increasing realization of the importance of knowledge of collagen at a fundamental level will almost certainly lead to the identification of the activating factors, the significance of the observed changes in type of collagen, and the nature of the cross-links and hence lead to more rational treatments. 